13 So PP-S, Davies RA, Chandy G, et al. Usefulness of $\beta$-blocker therapy and outcomes in patients with pulmonary arterial hypertension. Am J Cardiol 2012; 109: 1504-1509.

14 Thenappan T, Roy SS, Duval S, et al. $\beta$-blocker therapy is not associated with adverse outcomes in patients with pulmonary arterial hypertension: a propensity score analysis. Circ Heart Fail 2014; 7: 903-910.

15 Perros F, Ranchoux B, Izikki M, et al. Nebivolol for improving endothelial dysfunction, pulmonary vascular remodeling, and right heart function in pulmonary hypertension. J Am Coll Cardiol 2015; 65: 668-680.

16 Grinnan D, Bogaard H-J, Grizzard J, et al. Treatment of group I pulmonary arterial hypertension with carvedilol is safe. Am J Respir Crit Care Med 2014; 189: 1562-1564.

17 Martyniuk TV, Konosova ID, Chazova IE. [Use of nebivolol in patients with idiopathic pulmonary hypertension: results of the pilot study.] Ter Arkh 2012; 84: 49-53.

\title{
Cameroon's MDR-TB treatment programme jeopardised by cross-border migration
}

To the Editor:

We read with interest in a recent issue of the European Respiratory Journal, a paper by LÖNNROTH et al. [1] entitled. "Towards tuberculosis elimination: an action framework for low-incidence countries". The authors highlight cross-border migration as a major challenge for tuberculosis (TB) control efforts and their action framework pleads for strong international partnerships for reaching global TB elimination targets. To further comment on the challenges posed by cross-border migration, we here report on a recent experience of Cameroon's National TB Programme (NTP).

Since 2005, Cameroon's NTP has been treating multidrug-resistant (MDR)-TB patients with standardised therapeutic regimens inspired by the experiences of VAN DEUN and colleagues [2,3] in Bangladesh. From 2008 to 2012, patients were treated using a short duration regimen (12 months); a cohort assessment of the first 150 patients revealed an $89 \%$ therapeutic success rate [4]. Since 2013, patients have been treated using a 9-month regimen (4KmMfxPtoClzEZH/5MfxClzEZ; known as the "Bangladesh regimen"). Evaluation of a first cohort shows a therapeutic success rate of $82 \%$. In 2014, a total of 91 patients were put on treatment. Unlike the World Health Organization (WHO) recommended regimens for the management of drug-resistant $\mathrm{TB}$, short-course regimens are easily implemented, use less toxic drugs, are more tolerable, limit the use of injectables and reduce the risk of treatment interruption and defaulting [5, 6]. Moreover, the fact that patients in Cameroon have very little or no exposure to second-line anti-TB drugs, particularly to fluoroquinolones, for the treatment of diseases other than TB, might contribute to the programme success. How fluoroquinolone resistance in MDR-TB patients compromises favourable treatment outcomes was shown by FALzON et al. [7] in a large individual patient data meta-analysis.

For several years, MDR-TB patients from neighbouring countries not offering MDR-TB treatment, for example Equatorial Guinea, have been put on standardised treatment in Cameroon, based on the assumption that the epidemiological situation in these countries may possibly be similar to that of Cameroon. Since the beginning of 2015, Cameroon's NTP has seen a large influx of MDR-TB patients from Equatorial Guinea. 14 MDR-TB patients have been put on treatment on the basis of an Xpert MTB/RIF (Cepheid, Maurens-Scopont, France) test result "Rifampicin-resistance" in accordance with the national guidelines.

An early patient from Equatorial Guinea proved resistant to fluoroquinolones in late 2013 and eventually failed her treatment, and was considered as an "anecdotal" event. Recently, a second patient's cultures did not convert to negative after 6 months of treatment. Drug-resistance testing by line probe assay (LPA) once again brought to light resistance to fluoroquinolones. Interviews with patients revealed that most of them had been exposed to repetitive courses of ciprofloxacin (Cfx) for the treatment of typhoid fever, a pathology widely over-diagnosed and over-treated in Central Africa. Table 1 summarises epidemiological and clinical characteristics of the Equatorial Guinean patients put on treatment since 2013 with LPA results awaited for some of them.

Two practical questions arise immediately. Should the 9-month regimen of patients with a history of repetitive exposure to Cfx who are presently under treatment be changed and how? Also, how best could patients who do not convert be treated and where to perform the treatment? 
TABLE 1 Epidemiological and clinical data of patients from Equatorial Guinea put on standardised short-course MDR-TB treatment in Cameroon (2013-2015)

\begin{tabular}{|c|c|c|c|c|c|c|c|c|}
\hline Patient & $\begin{array}{l}\text { Treatment } \\
\text { start }\end{array}$ & Age & Sex & $\begin{array}{l}\text { Type of } \\
\text { patient }\end{array}$ & Cfx exposure ${ }^{\pi}$ & $\begin{array}{c}\text { Drug resistance } \\
\text { profile }^{+}\end{array}$ & $\begin{array}{l}\text { Results of LPA } \\
\text { (Hain } 囚 \text { ) testing for } \\
\text { resistance to } \mathrm{Flq}^{+}\end{array}$ & $\begin{array}{l}\text { Treatment } \\
\text { outcome }\end{array}$ \\
\hline 2 & 03 Nov 2013 & 53 & $\mathrm{~F}$ & $\mathrm{~F} 2$ & NA & RHEZ & Yes & Failure \\
\hline 3 & 11 Nov 2014 & 36 & $\mathrm{~F}$ & F2 & +++ & RHES & Yes & Failure \\
\hline 4 & 03 April 2015 & 26 & $M$ & F2 & No & RHE & Testing ongoing & Under treatment \\
\hline 7 & 22 April 2015 & 33 & $\mathrm{~F}$ & $\mathrm{~F} 2$ & +++ & $\mathrm{R}$ & No culture growth & Under treatment \\
\hline 8 & 22 April 2015 & 42 & M & F2 & No & RHE & No & Under treatment \\
\hline 9 & 22 April 2015 & 20 & $M$ & F2 & ++ & RHE & No & Under treatment \\
\hline 10 & 24 April 2015 & 29 & $M$ & F2 & +++ & RHE & No culture growth & Under treatment \\
\hline 11 & 24 April 2015 & 23 & $M$ & R1 & No & RHES & No & Under treatment \\
\hline 12 & 28 April 2015 & 32 & $M$ & F2 & ++ & RHES & No & Under treatment \\
\hline
\end{tabular}

Cfx: ciprofloxacin; LPA: line probe assay; Flq: fluoroquinolone; M: male; F: female; F1: Failure after first treatment; F2: failure after (several) retreatments; R1: relapse after first treatment; NA: not available; +: recalled one treatment episode; ++: recalled two treatment episodes; +++ recalled three or more treatment episodes; E: Ethambutol; H: isoniazid; O: ofloxacin; R: rifampicin; S: streptomycin.

Yet, this cross-border "invasion" of MDR-TB patients with hitherto unknown drug-resistant profiles from a neighbouring country with an apparently sub-optimally functioning NTP is worrying not only in practical terms. The management of drug-resistant TB in Cameroon was conceived and implemented following systematically gathered knowledge of the local endemic, continuous monitoring and careful evaluation. The programme functions thanks to entirely external funding (Global Fund) based on projected local endemic trends. The sudden and massive influx of patients with resistance profiles not taken into account when implementing the programme represents a heavy burden to the already limited resources and requires additional means (revised diagnostic and treatment protocols, training, procurement of additional drug etc.). At stake are also the programme's outcome indicators with failure, death and, in particular, lost to follow-up rates expected to increase. Ultimately, transmission and subsequent spread of fluoroquinolone-resistant MTB strains among the indigenous population should to be feared.

What next? Without delay, the NTPs of Equatorial Guinea and Cameroon should jointly assess the magnitude of the present MDR-TB problem in Equatorial Guinea. How many candidates for treatment are there, where should they be treated and under what conditions? Moreover, the performance of the NTP of Equatorial Guinea should be scrutinised, weaknesses identified and immediate and strong remedial measures taken. It would be worth considering a potentially very fruitful south-south collaboration under the aegis of the local WHO representations, supported by a technical north-south partnership, also in the interest of TB elimination in the north [8].

Finally, in 2014, participants of a symposium organised by the European Respiratory Society pleaded for the rational use of new and existing TB drugs [9]. And very recently, the Stop TB Partnership joined the call for urgent action of the G7 group of countries, highlighting antimicrobial resistance as a top priority on the global development agenda [10]. Indiscriminate prescription of broad-spectrum antibiotics like Cfx for doubtful pathologies, which still has to be confirmed for Equatorial Guinea, would be another example of the disastrous consequences of antibiotic misuse.

@ERSpublications

MDR-TB treatment programme can be jeopardised by cross-border migration as seen in Cameroon http://ow.ly/WHbxw

Jürgen Noeske ${ }^{1}$, Jean-Louis Abena Foe ${ }^{2}$ and Christopher Kuaban ${ }^{3,4}$

${ }^{1}$ Senior Consultant, National Tuberculosis Programme, Yaounde, Cameroon. ${ }^{2}$ National Tuberculosis Programme, Yaounde, Cameroon. ${ }^{3}$ Faculty of Medicine and Biomedical Sciences, University of Yaounde, Yaounde, Cameroon.

${ }^{4}$ Faculty of Health Sciences, University of Bamenda, Bamenda, Cameroon. 
Correspondence: Jürgen Noeske, NTP, Yaounde, Cameroon. E-Mail: juergennoeske@yahoo.fr

Received: Aug 102015 | Accepted after revision: Sept 022015

Conflict of interest: None declared

References

1 Lönnroth K, Migliori GB, Abubakar I, et al. Towards tuberculosis elimination: an action framework for low-incidence countries. Eur Respir J 2015; 45: 928-952.

2 Van Deun A, Salim MA, Das AP, et al. Results of a standardized regimen for multidrug-resistant tuberculosis in Bangladesh. Int J Tuberc Lung Dis 2004; 8: 560-567.

3 Van Deun A, Maug AK, Salim MA, et al. Short, highly effective, and inexpensive standardized treatment of multidrug-resistant tuberculosis. Am J Respir Crit Care Med 2010; 182: 684-692.

4 Kuaban C, Noeske J, Rieder H, et al. High effectiveness of a 12-month regimen for MDR-TB patients in Cameroon. Int J Tuberc Lung Dis 2015; 19: 517-524.

5 Falzon D, Jaramillo E, Schünemann HJ, et al. WHO guidelines for the programmatic management of drug-resistant tuberculosis: 2011 update. Eur Respir J 2011; 38: 516-528.

6 Chiang C-Y, Van Deun A, Enarson DA. A poor drug-resistant tuberculosis programme is worse than no programme: time for a change. Int J Tuberc Lung Dis 2013; 17: 714-718.

7 Falzon D, Gandhi N, Migliori GB, et al. Resistance to fluoroquinolones and second-line injectable drugs: impact on MDR-TB outcomes. Eur Respir J 2013; 42: 156-168.

8 D'Ambrosio L, Dara M, Tadolini M, et al. Tuberculosis elimination: theory and practice in Europe. Eur Respir J 2014; 43: 1410-1420.

9 Migliori GB, Lienhardt C, Weyer K, et al. Outcome of an ERS multisectoral consultation. Eur Respir J 2014; 44: $1412-1417$.

10 Stop TB Partnership. Stop TB Partnership calls for Urgent Action on Antimicrobial Resistance at the United Nations in New York.. Available from http://tbonline.info/posts/2015/6/21/stop-tb-partnership-calls-urgent-action-antimicrob/ Date last updated: June 21, 2015. Date last accessed: Oct 30, 2015.

Eur Respir J 2016; 47: 684-686 | DOI: 10.1183/13993003.01324-2015 | Copyright @eERS 2016

\section{From the authors:}

We read with interest the correspondence from J. Noeske and colleagues on the potential effect of cross-border migration on the multidrug-resistant tuberculosis (MDR-TB) treatment programme in Cameroon, elicited by our article on the framework for TB elimination in low TB incidence countries [1]. The manuscript highlights some of the consequences of cross-border migration for caring for people with TB and ending this epidemic, as well as relevant issues on clinical and public health management of MDR-TB in Cameroon.

Cross-border migration significantly contributes to TB epidemiology in many low incidence countries in Europe and elsewhere (figure 1) [3].

The effect is higher in countries that are close to the pre-elimination threshold, mainly since these countries are quite often attractive destinations due to a social environment more favourable to the needs of the migrants. In some low-incidence countries cross-border migration caused either by political, economic or environmental reasons is already becoming the main challenge to countries well advanced in the elimination of TB.

The vision of TB elimination is a global one: it should be immediately pursued in low TB incidence countries, and their experience should hopefully inspire action in countries with higher TB incidence.

As in the Cameroon example, people may migrate to access TB care of perceived higher quality or because in their original settings services are absent [4]. Others may voluntarily leave the host country during TB treatment, while some immigrants (in conflict with basic human rights and public health principles) are deported at the time of diagnosis or during treatment. In such cases, insufficient coordination of TB care services across borders or limited capacity at the receiving end can lead to a low quality of care (discontinuation of case holding), continued transmission and incomplete TB surveillance [5]. As pointed out by Noeske and colleagues, migration in search of quality healthcare may happen specifically to obtain second-line TB treatment which is not available in the country of origin. Programmes in the country where the diagnosis is made have the ethical imperative to treat patients until cure or to ensure that treatment is provided until the patient is cured [3]. Noeske and colleagues warn about the risk that a flow of migrants affected by drug-resistant TB would pose to the efforts of the host country to end TB and MDR-TB, although little evidence of transmission is available, particularly in Africa where more studies are needed. The authors advocate for joint measures across borders to contain the risk. Even in low incidence countries the influx of migrants with highly resistant forms of TB has already translated into challenges for contact investigation efforts, outbreak management and surveillance [6]. The same would 